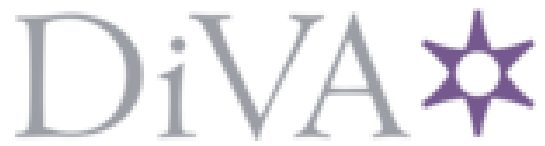

http://www.diva-portal.org

Preprint

This is the submitted version of a paper presented at IEEE Conference on Decision and Control (CDC'18).

Citation for the original published paper:

Mattila, R., Rojas, C R., Krishnamurthy, V., Wahlberg, B. (2018)

Inverse Filtering for Linear Gaussian State-Space Models

In: Proceedings of the 57th IEEE Conference on Decision and Control (CDC'18), Miami

Beach, FL, USA, 2018.

N.B. When citing this work, cite the original published paper.

Permanent link to this version:

http://urn.kb.se/resolve?urn=urn:nbn:se:kth:diva-277031 


\title{
Inverse Filtering for Linear Gaussian State-Space Models
}

\author{
Robert Mattila*, Cristian R. Rojas*, Vikram Krishnamurthy ${ }^{\dagger}$ and Bo Wahlberg*
}

\begin{abstract}
This paper considers inverse filtering problems for linear Gaussian state-space systems. We consider three problems of increasing generality in which the aim is to reconstruct the measurements and/or certain unknown sensor parameters, such as the observation likelihood, given posteriors (i.e., the sample path of mean and covariance). The paper is motivated by applications where one wishes to calibrate a Bayesian estimator based on remote observations of the posterior estimates, e.g., determine how accurate an adversary's sensors are. We propose inverse filtering algorithms and evaluate their robustness with respect to noise (e.g., measurement or quantization errors) in numerical simulations.
\end{abstract}

\section{INTRODUCTION}

In a partially observed Markovian model, the state of the system is latent and can only be indirectly observed via a noisy sensor. This model class includes, for example, the discrete hidden Markov model (HMM, e.g., [1]-[3]) and the linear Gaussian state-space model (e.g., [4], [5]). The classical stochastic filtering problem deals with estimating the latent state at the present time, given noisy observations up to the present time. For discrete HMMs, the optimal filter is the so-called HMM filter [1], [2], and for linear Gaussian state-space models, the solution is the Kalman filter [4].

In this paper, we consider several inverse filtering problems for linear Gaussian state-space models. In comparison to the standard filtering problem, which seeks to compute the posterior and conditional mean estimates of the state given noisy observations, our aims are:

Given posteriors from a Kalman filter, can we reconstruct

i) its sensor specifications?

ii) the noisy observations (measurements) that were made?

Affirmative answers to these questions have a number of potential applications; for example, fault detection and diagnosis (with several data batches, change detection can be performed on the reconstructed sensor specifications to see if a potential failure has occurred), reverse engineering of integrated sensor systems, electronic warfare (estimating the accuracy of an enemy's sensors), cyberphysical security, and autonomous calibration of remote sensors.

We provide answers to these questions by studying three inverse filtering problems of increasing order of generality.

This work was partially supported by the Swedish Research Council under contract 2016-06079 and the U.S. Air Force Office of Scientific Research under grant FA9550-18-1-0007. ${ }^{*}$ Robert Mattila, Cristian R. Rojas and Bo Wahlberg are with the Department of Automatic Control, School of Electrical Engineering and Computer Science, KTH Royal Institute of Technology, Stockholm, Sweden. (e-mails: rmattila,crro,bo@kth.se). †Vikram Krishnamurthy is with the Department of Electrical and Computer Engineering and Cornell Tech, Cornell University, NY, USA. (e-mail: vikramk@cornell.edu).
First: given sensor specifications and posterior distributions from a Kalman filter, reconstruct the sequence of observations that was processed. Second: given the observations that were processed and the posterior distributions, reconstruct sensor specifications. Third, the combined problem: given only the posterior distributions, what can be said regarding the sensor and the observations? Throughout, to limit the scope of the paper, we assume that the system dynamics are known to us (which is valid, for example, when we have constructed the system and someone else has built the sensor equipment that we aim to reverse engineer).

In summary, the three-fold contributions of this paper are:

- We consider the three problems stated above and propose inverse filtering algorithms to solve them.

- We demonstrate how the algorithms should be modified to account for noise (originating, for example, from realworld quantization and measurement errors).

- We illustrate the performance of the proposed inverse filtering algorithms via numerical examples.

The outline of the paper is as follows. Below, in Section IA, we give a brief overview of related work. In Section II, we detail the notation used in this paper, as well as necessary preliminaries related to the Kalman filter. In Section III, the inverse filtering problems are formally posed, and our proposed solutions are described in Section IV. The algorithms are illustrated on numerical examples in Section VI.

\section{A. Related work}

The study of inverse problems has a long history in the field of control. Kalman studied the problem of inverse optimal control already in 1964 [6], aiming to determine for what cost criteria a given control policy is optimal. More recently, inverse problems have sparked interest in the machine learning community under fields such as inverse reinforcement learning, imitation learning and apprenticeship learning [7]-[11]. In these frameworks, an agent tries to learn how to control a system by observing an expert (teacher) demonstrating the task to be performed. Related models are also used in economics under the umbrella of social learning [1] - in these, agents seek to estimate the underlying state given noisy measurements of the posterior from previous agents.

More specifically, inverse filtering problems have previously been posed in fault detection settings, where the Kalman filter is a classical tool (e.g., [12]-[15]). Typically, the corresponding tests are based on the residuals

$$
y_{k}-C^{T} \hat{x}_{k}
$$


and their variances, where ${ }^{T}$ denotes transpose, $y_{k}$ is the observation made at time $k, C$ is the sensor matrix and $\hat{x}_{k}$ is the Kalman state estimate (see equations (2b) and (4) below). For integrated smart sensors, it may be difficult to access the raw measurements $y_{k}$ and/or the $C$ matrix. Hence, a relevant question is: Can the residuals be reconstructed from the estimate $\hat{x}_{k}$ and its associated covariance matrix?

Initial work on this problem can be found in [16], [17]. The motivating application in these papers is mobile robots, where the pose (position and orientation) of a robot is an important quantity that has to be estimated. The pose can indirectly be measured from several different sensor systems such as odometry, computer vision, sonar and laser. The output from these so-called pose providers are often state estimates together with, in best cases, an error covariance matrix estimate. It may be very difficult, or even impossible, to access the raw sensor data since the sensor and state estimator/observer often are integrated and encapsulated. In [16], [17], an extended observer was used to reconstruct the residuals (1). In contrast, we take a more direct approach and aim to reconstruct the quantities in the expression (1) explicitly.

It was recently shown how questions similar to those posed in the introduction can be (affirmatively) answered for the HMM filter [18]. In particular, [18] shows that both sensor specifications and the sample path of observations can be reconstructed from posteriors. Moreover, the reconstruction procedure can efficiently exploit the algebraic structure of the HMM filter so as to avoid computationally expensive formulations based on combinatorial optimization. The present paper can be seen as an extension of the results in [18] to linear Gaussian state-space models.

\section{PREliminaries}

In this section, we detail the notation, the system model and state the Kalman filter recursion.

\section{A. Notation}

Vectors are column vectors, unless transposed. $\|\cdot\|_{2}$ denotes the Euclidean norm of a vector. The symbol $\sim$ is short for "distributed according to". The Gaussian (normal) distribution with mean vector $\mu$ and covariance matrix $\Sigma$ is denoted $\mathcal{N}(\mu, \Sigma)$. We denote the maximum modulus eigenvalue of a matrix as $\lambda_{\max }(\cdot)$.

\section{B. System model}

We consider (potentially time-varying) autonomous linear state-space systems with Gaussian noise:

$$
\begin{aligned}
x_{k+1} & =A_{k} x_{k}+w_{k}, \\
y_{k} & =C^{T} x_{k}+v_{k},
\end{aligned}
$$

where $x_{k} \in \mathbb{R}^{X}$ is the state vector at time $k, y_{k} \in \mathbb{R}^{Y}$ is the observation made at time $k, w_{k} \sim \mathcal{N}\left(0, Q_{k}\right)$ is the process noise and $v_{k} \sim \mathcal{N}(0, R)$ is the sensor noise. We assume that $\left\{w_{k}\right\}$ and $\left\{v_{k}\right\}$ are independent of each other, and also between samples. We allow for a time-varying plant (via $A_{k}$ and $Q_{k}$ ), but restrict to a time-invariant sensor (via $C$ and $R$ ).
In order to limit the scope of the paper, we consider only single-output systems, i.e., $Y=1$. For this setting, we define the signal-to-noise ratio $\eta$ of the sensor system as

$$
\eta \stackrel{\text { def. }}{=} \frac{\|C\|_{2}}{\sqrt{R}} .
$$

\section{Kalman filter}

For the system model (2a)-(2b), it is well-known (e.g., [1], [4]) that, under standard assumptions, the posterior density

$$
\pi_{k}(x)=p\left(x_{k}=x \mid y_{1}, \ldots, y_{k}\right)
$$

has a finite-dimensional characterisation

$$
\pi_{k} \sim \mathcal{N}\left(\hat{x}_{k}, \Sigma_{k}\right)
$$

which can be computed via the classical Kalman filter equations:

$$
\begin{aligned}
\hat{x}_{k+1 \mid k} & =A_{k} \hat{x}_{k}, \\
y_{k+1 \mid k} & =C^{T} \hat{x}_{k+1 \mid k}, \\
\Sigma_{k+1 \mid k} & =A_{k} \Sigma_{k} A_{k}^{T}+Q_{k}, \\
S_{k+1} & =C^{T} \Sigma_{k+1 \mid k} C+R, \\
\hat{x}_{k+1} & =\hat{x}_{k+1 \mid k}+\Sigma_{k+1 \mid k} C S_{k+1}^{-1}\left(y_{k+1}-y_{k+1 \mid k}\right), \\
\Sigma_{k+1} & =\Sigma_{k+1 \mid k}-\Sigma_{k+1 \mid k} C S_{k+1}^{-1} C^{T} \Sigma_{k+1 \mid k},
\end{aligned}
$$

where a Gaussian prior $\pi_{0} \sim \mathcal{N}\left(\hat{x}_{0}, \Sigma_{0}\right)$ has been assumed.

\section{PROBLEM Formulation}

The system (plant process) is characterised by (2a), whereas the sensor used to measure it is characterised by (2b). In this paper, we consider inverse filtering problems in which the system dynamics are known, and we aim to reconstruct specifications of the sensor. Note that, to limit the scope of the paper, we do not consider mismatched filtering problems (that is, when the true plant is different from the plant model).

We consider the following three inverse filtering problems that are stated in an increasing order of generality:

Problem 1 (Inverse filtering with unknown observations). Assume that the system dynamics $\left\{A_{k}, Q_{k}\right\}_{k}$ in (2a) are known, as well as the sensor characteristics $\{C, R\}$ in (2b). A Kalman filtering system provides us with posterior state distributions characterised by $\left\{\hat{x}_{k}, \Sigma_{k}\right\}_{k}$. Reconstruct the observations $\left\{y_{k}\right\}_{k}$ that were processed.

In essence, the first problem involves reconstructing the sample path of observations, when both the characteristics of the system and the sensor are known. The second problem is the complementary problem to this:

Problem 2 (Inverse filtering with unknown sensor). Assume that the system dynamics $\left\{A_{k}, Q_{k}\right\}_{k}$ in (2a) are known, as well as the observations $\left\{y_{k}\right\}_{k}$ that were sampled by the sensor. A Kalman filtering system provides us with posterior state distributions characterised by $\left\{\hat{x}_{k}, \Sigma_{k}\right\}_{k}$. Reconstruct the sensor characteristics $\{C, R\}$ in $(2 b)$. 


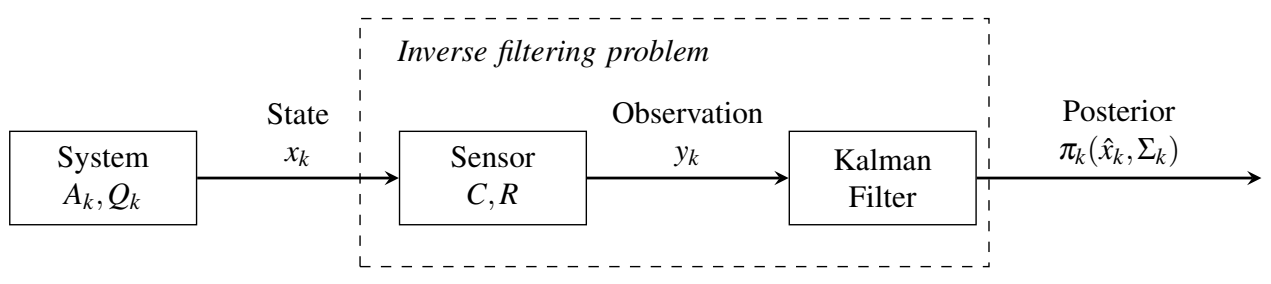

Fig. 1: Overview of the inverse filtering problem setup. The system updates the state $x_{k}$ according to the system dynamics $\left\{A_{k}, Q_{k}\right\}$. An observation $y_{k}$ is sampled using the sensor according to its specifications $\{C, R\}$. This observation is processed by the Kalman filter which computes a new posterior distribution $\pi_{k}$, parametrized by $\left\{\hat{x}_{k}, \Sigma_{k}\right\}$. In the inverse filtering problem (Problem 3), we aim to reconstruct the sensor specifications $\{C, R\}$, as well as the sample path of observations $\left\{y_{k}\right\}_{k}$ from a known system $\left\{A_{k}, Q_{k}\right\}_{k}$ and posterior readings $\left\{\hat{x}_{k}, \Sigma_{k}\right\}_{k}$.

Finally, we consider the general setting, which is the combination of the two previous problems; both the sample path of the observation process and the sensor are unknown to us.

Problem 3 (Inverse filtering with unknown sensor and observations). Assume that the system dynamics $\left\{A_{k}, Q_{k}\right\}_{k}$ in (2a) are known. A Kalman filtering system provides us with posterior state distributions characterised by $\left\{\hat{x}_{k}, \Sigma_{k}\right\}_{k}$. Reconstruct the sensor characteristics $\{C, R\}$ in $(2 b)$, and the observations $\left\{y_{k}\right\}_{k}$ that were processed.

These are the three nominal problems that we will discuss in this paper. A schematic overview of the setup is given in Fig. 1, together with an explanation of Problem 3. In Section V, we will consider reformulations of the problems to the more general setting where uncertainty is present on the posterior data.

\section{Algorithms For InVERSE Filtering}

In this section, we outline solutions to Problems 1-3 that were stated in the previous section.

\section{A. Solution to Problem 1}

In the first problem, we are asked to reconstruct the observations that were processed by the filtering system. This can be accomplished as follows:

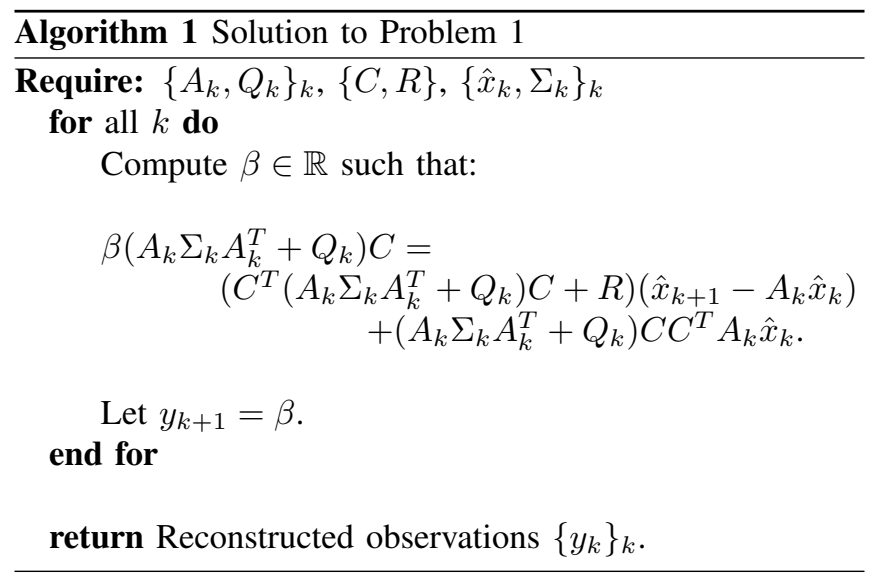

Theorem 1. If $\left(A_{k} \Sigma_{k} A_{k}^{T}+Q_{k}\right) C \neq 0$ for all $k$, then the sequence of observations can be reconstructed exactly using Algorithm 1.

Proof. By simple manipulations of (6e), we obtain

$$
\begin{array}{r}
S_{k+1}\left(\hat{x}_{k+1}-\hat{x}_{k+1 \mid k}\right)+\Sigma_{k+1 \mid k} C y_{k+1 \mid k} \\
=\Sigma_{k+1 \mid k} C y_{k+1} .
\end{array}
$$

From this, we conclude that $y_{k+1}$ can be obtained as the scaling factor of the vector in the left-hand side (LHS) with respect to the vector in the right-hand side (RHS) $\Sigma_{k+1 \mid k} C$. By introducing (6a)-(6c) in (7), we can express this explicitly in terms of only the given quantities. The vector in the RHS becomes

$$
\left(A_{k} \Sigma_{k} A_{k}^{T}+Q_{k}\right) C
$$

and the LHS becomes

$$
\begin{aligned}
\left(C^{T}\left(A_{k} \Sigma_{k} A_{k}^{T}+Q_{k}\right) C\right. & +R)\left(\hat{x}_{k+1}-A_{k} \hat{x}_{k}\right) \\
& +\left(A_{k} \Sigma_{k} A_{k}^{T}+Q_{k}\right) C C^{T} A_{k} \hat{x}_{k} .
\end{aligned}
$$

Equation (7) is a set of $X$ equations for one unknown variable $\left(y_{k+1}\right)$. The true parameters are, of course, consistent with the equations, which guarantees the existence of a solution. Moreover, the solution is unique unless the RHS vector is zero.

\section{B. Solution to Problem 2}

In the formulation of the second problem, we assume that we have access to the observations $\left\{y_{k}\right\}_{k}$ made by the sensor, but that its specifications $\{C, R\}$ are unknown and to be reconstructed. This can be done as follows:

Theorem 2. If, for some $k,\left(A_{k} \Sigma_{k} A_{k}^{T}+Q_{k}\right)$ is non-singular and $y_{k+1} \neq 0$, then the sensor specifications $\{C, R\}$ can be reconstructed exactly using Algorithm 2.

Proof. By introducing equations (6c) and (6d) in equation (6f), we obtain

$$
\begin{gathered}
\Sigma_{k+1}=\left(A_{k} \Sigma_{k} A_{k}^{T}+Q_{k}\right)-\left(A_{k} \Sigma_{k} A_{k}^{T}\right. \\
\left.+Q_{k}\right) C\left[C^{T}\left(A_{k} \Sigma_{k} A_{k}^{T}+Q_{k}\right) C+R\right]^{-1} C^{T}\left(A_{k} \Sigma_{k} A_{k}+Q_{k}\right),
\end{gathered}
$$


which we can rewrite as

$$
\begin{gathered}
{\left[I-\left(A_{k} \Sigma_{k} A_{k}^{T}+Q_{k}\right)^{-1} \Sigma_{k+1}\right]\left(A_{k} \Sigma_{k} A_{k}^{T}+Q_{k}\right)^{-1}} \\
=C\left[C^{T}\left(A_{k} \Sigma_{k} A_{k}^{T}+Q_{k}\right) C+R\right]^{-1} C^{T} .
\end{gathered}
$$

To ease the notation, denote the LHS of the previous equation as

$$
\Omega \stackrel{\text { def. }}{=}\left[I-\left(A_{k} \Sigma_{k} A_{k}^{T}+Q_{k}\right)^{-1} \Sigma_{k+1}\right]\left(A_{k} \Sigma_{k} A_{k}^{T}+Q_{k}\right)^{-1} .
$$

Note that the matrix $\Omega$ is known to us since all quantities in its definition (12) are given.

Continuing, let

$$
C=\alpha \bar{C},
$$

where $\alpha \in \mathbb{R}$ and $\|\bar{C}\|_{2}=1$. Then, by noting that $C^{T}\left(A_{k} \Sigma_{k} A_{k}^{T}+Q_{k}\right) C+R$ in equation (11) is scalar, we have that:

$$
\begin{aligned}
\Omega & =C\left[C^{T}\left(A_{k} \Sigma_{k} A_{k}^{T}+Q_{k}\right) C+R\right]^{-1} C^{T} \\
& =\frac{C C^{T}}{C^{T}\left(A_{k} \Sigma_{k} A_{k}^{T}+Q_{k}\right) C+R} \\
& =\frac{\alpha^{2}}{\alpha^{2} \bar{C}^{T}\left(A_{k} \Sigma_{k} A_{k}^{T}+Q_{k}\right) \bar{C}+R} \cdot \bar{C} \bar{C}^{T} .
\end{aligned}
$$

This equation shows that $\Omega$ is a rank-one matrix. By performing a spectral decomposition ${ }^{1}$ of $\Omega$, we obtain i) $\bar{C}$ as the eigenvector corresponding to the non-zero eigenvalue $\lambda_{\max }(\Omega)$, and $\left.i i\right)$ that

$$
\lambda_{\max }(\Omega)=\frac{\alpha^{2}}{\alpha^{2} \bar{C}^{T}\left(A_{k} \Sigma_{k} A_{k}^{T}+Q_{k}\right) \bar{C}+R} .
$$

To compute the factor $\alpha$ in relation (13), introduce equations (6b)-(6d) in equation (6e) as follows:

$$
\begin{aligned}
\hat{x}_{k+1}-\hat{x}_{k+1 \mid k} & =\frac{\Sigma_{k+1 \mid k} C\left(y_{k+1}-y_{k+1 \mid k}\right)}{C^{T} \Sigma_{k+1 \mid k} C+R} \\
& =\frac{\left(A_{k} \Sigma_{k} A_{k}^{T}+Q_{k}\right) C\left(y_{k+1}-C^{T} \hat{x}_{k+1 \mid k}\right)}{C^{T}\left(A_{k} \Sigma_{k} A_{k}^{T}+Q_{k}\right) C+R} .
\end{aligned}
$$

This can be further rewritten using (6a) as

$$
\begin{aligned}
& \left(A_{k} \Sigma_{k} A_{k}^{T}+Q_{k}\right)^{-1}\left(\hat{x}_{k+1}-A_{k} \hat{x}_{k}\right) \\
& \quad=\frac{C}{C^{T}\left(A_{k} \Sigma_{k} A_{k}^{T}+Q_{k}\right) C+R}\left(y_{k+1}-C^{T} A_{k} \hat{x}_{k}\right) .
\end{aligned}
$$

Again, to simplify the notation, denote the LHS by

$$
\Psi \stackrel{\text { def. }}{=}\left(A_{k} \Sigma_{k} A_{k}^{T}+Q_{k}\right)^{-1}\left(\hat{x}_{k+1}-A_{k} \hat{x}_{k}\right),
$$

and let the coefficient vector of the RHS be

$$
\Upsilon \stackrel{\text { def. }}{=} \frac{C}{C^{T}\left(A_{k} \Sigma_{k} A_{k}^{T}+Q_{k}\right) C+R} \text {. }
$$

By the first equality in equation (14), we have that

$$
\Omega=\Upsilon C^{T},
$$

\footnotetext{
${ }^{1}$ This can be computed efficiently by taking any non-zero column of $\Omega$ and normalizing it to have unit norm. From there the eigenvalue can easily be computed.
}

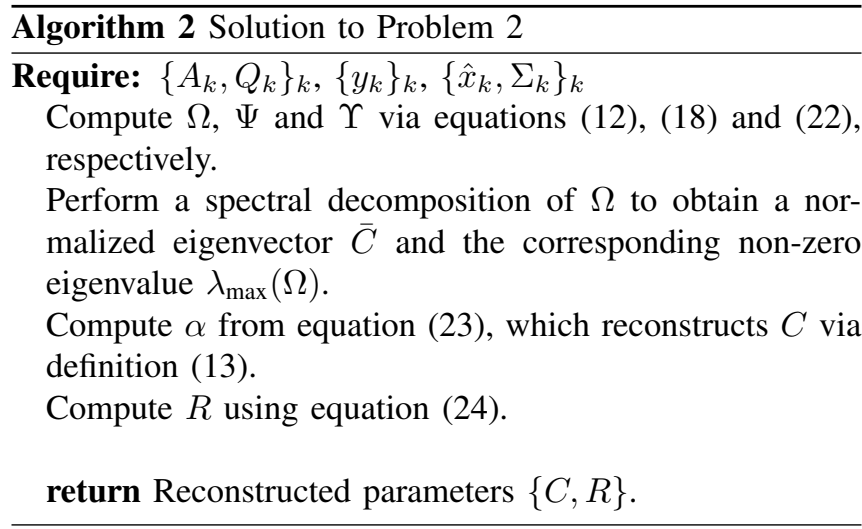

so that equation (17) can be concisely expressed as

$$
\begin{aligned}
\Psi & =\Upsilon\left(y_{k+1}-C^{T} A_{k} \hat{x}_{k}\right) \\
& =\Upsilon y_{k+1}-\Omega A_{k} \hat{x}_{k} .
\end{aligned}
$$

From expression (21), we can solve for $\Upsilon$ as

$$
\Upsilon=\frac{1}{y_{k+1}}\left(\Psi+\Omega A_{k} \hat{x}_{k}\right),
$$

where everything on the RHS is known: $y_{k+1}$ by assumption, $\Omega$ from equation (12), and $\Psi$ from equation (18). Finally, by introducing definition (13) in (20);

$$
\Omega=\alpha \Upsilon \bar{C}^{T},
$$

we obtain $\alpha$ since $\Upsilon, \bar{C}$ and $\Omega$ are known. The existence of a solution $\alpha$ is guaranteed since the true parameters fulfill the equations.

To obtain $R$, we solve for it in equation (15):

$$
R=\frac{\alpha^{2}}{\lambda_{\max }(\Omega)}-\alpha^{2} \bar{C}^{T}\left(A_{k} \Sigma_{k} A_{k}^{T}+Q_{k}\right) \bar{C},
$$

which determines all unknown quantities of Problem 2.

\section{Solution to Problem 3}

In the most general case (Problem 3), both the sequence of observations and the sensor specifications are unknown to us. Since we do not have an absolute reference point for the gain of the observations, we will see that we can only identify them up to a scaling factor. ${ }^{2}$ As in equation (13) of the previous section, let

$$
C=\alpha \bar{C},
$$

where $\alpha \in \mathbb{R}$ and $\|\bar{C}\|_{2}=1$, then, in particular, we have that:

Theorem 3. If, for all $k,\left(A_{k} \Sigma_{k} A_{k}^{T}+Q_{k}\right)$ is non-singular, then:

- the signal-to-noise ratio (3), i.e.,

$$
\eta=\frac{\|C\|_{2}}{\sqrt{R}}=\frac{|\alpha|}{\sqrt{R}}
$$

\footnotetext{
${ }^{2}$ This parallels the corresponding result in [18], where the observations and sensor parameters of the discrete HMM can only be determined up to permutations.
} 
- the observations (up to a scaling factor),

- and the vector $\bar{C}$ (up to its sign),

can be reconstructed using Algorithm 3.

Proof. First, we follow the steps leading up to equation (15) in the solution to Problem 2. This determines the vector $\bar{C}$ (up to its sign). Next, from equation (15), it is easy to show that the ratio $\eta$ equals ${ }^{3}$

$$
\frac{|\alpha|}{\sqrt{R}}=\frac{1}{\sqrt{1 / \lambda_{\max }(\Omega)-\bar{C}^{T}\left(A_{k} \Sigma_{k} A_{k}^{T}+Q_{k}\right) \bar{C}}},
$$

where the RHS is known to us.

Finally, in order to obtain the observations, employ that $R=\alpha^{2} / \eta^{2}$ in equations (7)-(9), together with $C=\alpha \bar{C}$ :

$$
\begin{aligned}
\left(A_{k} \Sigma_{k} A_{k}^{T}\right. & \left.+Q_{k}\right) \bar{C}\left(\frac{y_{k+1}}{\alpha}\right)= \\
& \left(\bar{C}^{T}\left(A_{k} \Sigma_{k} A_{k}^{T}+Q_{k}\right) \bar{C}+\frac{1}{\eta^{2}}\right)\left(\hat{x}_{k+1}-A_{k} \hat{x}_{k}\right) \\
& +\left(A_{k} \Sigma_{k} A_{k}^{T}+Q_{k}\right) \bar{C} \bar{C}^{T} A_{k} \hat{x}_{k} .
\end{aligned}
$$

From this equation, it can be seen that $\frac{y_{k+1}}{\alpha}$ is determined as the scaling factor between the vector on the LHS with respect to that in the RHS. (Note that everything except $y_{k+1}$ and $\alpha$ is known to us.) The existence of a solution is guaranteed since the true parameters satisfy the equations. Moreover, the solution is unique as long as $\left(A_{k} \Sigma_{k} A_{k}^{T}+Q_{k}\right) \bar{C} \neq 0$, which holds since $\left(A_{k} \Sigma_{k} A_{k}^{T}+Q_{k}\right)$ is non-singular.

\section{Algorithms For InVERse Filtering With NOISE-CORRUPTED POSTERIORS}

In this section, we study the influence of uncertainties in the parameters on the inverse filtering problem. In particular, we consider the case when the parameters $\left\{\hat{x}_{k}, \Sigma_{k}\right\}_{k}$ of the posteriors are not known exactly, but rather are measured in noise. The motivation stems from electronic warfare; the enemy's sensors' measurements result in the enemy taking an action which can be viewed as a noisy function of its posterior. Based on this action, our goal is to calibrate the enemy's sensors. Formally, the generalization of Problem 3 the other problems follow as special cases - that we consider is:

Problem 4 (Inverse filtering in noise with unknown sensor and observations). Assume that the system dynamics $\left\{A_{k}, Q_{k}\right\}_{k}$ in (2a) are known. A Kalman filtering system provides posterior state distributions characterised by $\left\{\hat{x}_{k}, \Sigma_{k}\right\}_{k}$. From noisy versions $\left\{\bar{x}_{k}, \bar{\Sigma}_{k}\right\}_{k}$ of these signals, reconstruct the sensor characteristics $\{C, R\}$ in $(2 b)$, and the observations $\left\{y_{k}\right\}_{k}$ that were processed.

\section{A. Solution to Problem 4}

If the versions of posterior parameters $\left\{\hat{x}_{k}, \Sigma_{k}\right\}_{k}$ that we obtain are corrupted by noise, then the equations leading up to equation (15) will no longer hold with equality. This

\footnotetext{
${ }^{3}$ Note from this that it is enough that $\left(A_{k} \Sigma_{k} A_{k}^{T}+Q_{k}\right)$ is non-singular for some $k$ in order to reconstruct $\eta$ and $\bar{C}$.
}

implies that $\Omega$, as defined in (12), will no longer be a rankone matrix (as was shown to be the case in equation (14) for the noise-free setting).

However, by assuming that the perturbations are small, we can argue (by the continuity of its eigenvalues) that it will be close to a rank-one matrix. Hence, we can obtain an approximate solution by employing Algorithm 3 (with the only difference is that there will be multiple non-zero eigenvalues of $\Omega$ ).

It should be noted that in the noise-free case, we could recover the signal-to-noise ratio $\eta$ exactly from any time instant (where the matrix $\left(A_{k} \Sigma_{k} A_{k}^{T}+Q_{k}\right)$ was invertible). With noisy parameters, we will reconstruct a noisy estimate of $\eta$ for every time instant. It is hence reasonable to average the estimated $\eta$ :s over a sequence of times, instead of relying on the estimate from a single time instant.

\section{NUMERICAL SimUlations}

In this section, we illustrate the proposed inverse filtering algorithms on numerical data. We consider the following time-varying system

$$
\begin{aligned}
x_{k+1} & =\left[\begin{array}{ccc}
1.1269 & -0.4940 & 0.1129 \\
1.0000 & 0 & 0 \\
0 & 1.0000 & 0
\end{array}\right] x_{k}+w_{k}, \\
y_{k} & =\left[\begin{array}{lll}
0.5 & 0 & 0
\end{array}\right] x_{k}+v_{k},
\end{aligned}
$$

where the noise parameters are $R=10 ; Q_{k}=20 I$ if $k \in$ $\{10 d, 10 d+1, \ldots, 10 d+9: d=0,2,4, \ldots\}$ and $Q_{k}=100 I$ otherwise - that is, the process' noise level switches between two levels every 10 samples. The signal-to-noise ratio (3) of the sensor is $\eta \approx 0.1581$. In the simulations, we employ Algorithm 3, taking the discussion in Section V into account.

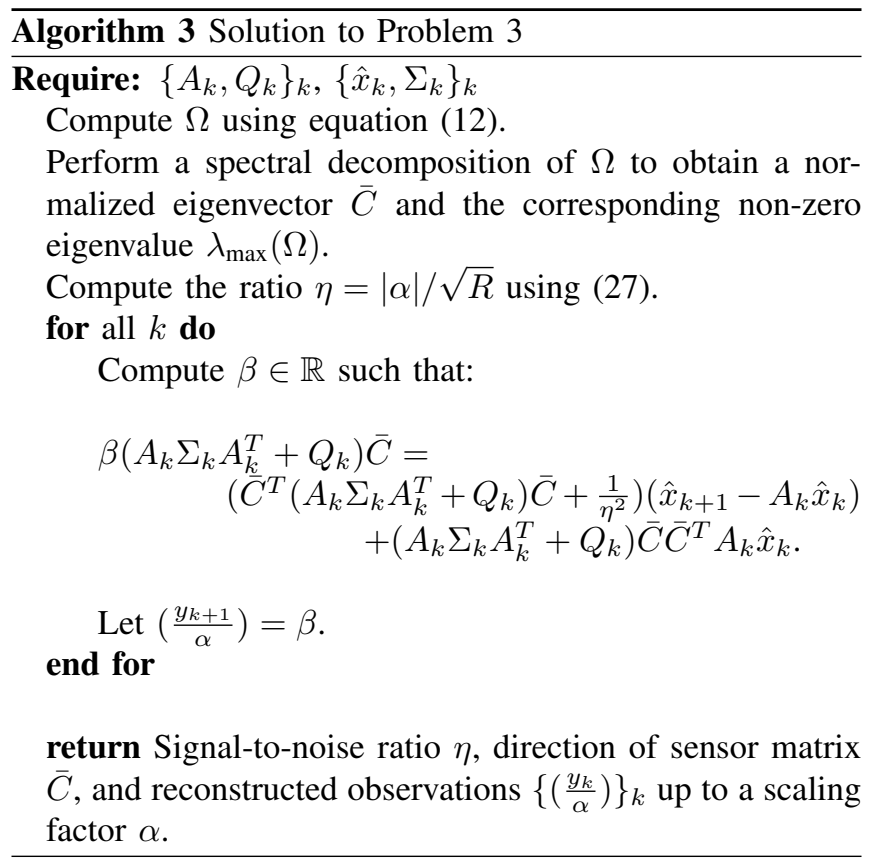


Residuals reconstructed via inverse filtering

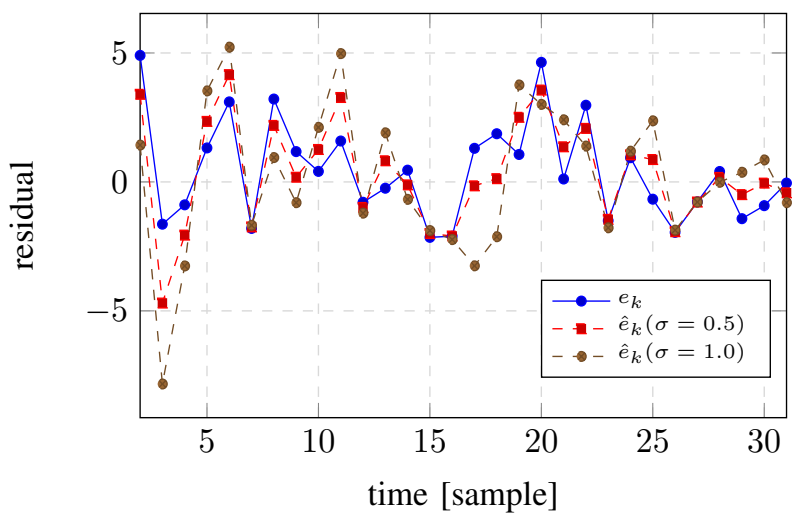

Fig. 2: The residuals $e_{k}=y_{k}-C^{T} \hat{x}_{k}$ and their reconstructions via inverse filtering. The posterior parameters $\hat{x}_{k}$ and $\Sigma_{k}$ have been perturbed elementwise with Gaussian noise of varying standard deviation $\sigma$.

\section{A. Measurement noise and fault-detection}

In model-based fault-detection, the statistics of the residuals between the system outputs $y_{k}$ and the model predictions $C^{T} \hat{x}_{k}$ are commonly studied (e.g., [12]-[15]). In practice, however, the measurement process can be tightly encapsulated in the sensor system, so that the measurements and sensor specifications are not directly available. An important problem is then to reconstruct the residuals from only posteriors from the filtering system (see, e.g., [16], [17], for more background). These residuals can be expressed as:

$$
\begin{aligned}
e_{k} & =y_{k}-C^{T} \hat{x}_{k} \\
& =\alpha\left[\left(\frac{y_{k}}{\alpha}\right)-\bar{C}^{T} \hat{x}_{k}\right],
\end{aligned}
$$

where everything in the square-bracket can be reconstructed using Algorithm 3. In particular, this means that the residuals can be reconstructed up to a scaling factor.

The scaling factor $\alpha$ is unknown to us, however, for change detection purposes, we can arbitrarily choose it as any non-zero constant. For illustratory purposes, we use its true value (since the simulated system (29) is known to us). The true sequence of residuals, along with its reconstruction after noise has been injected on the posterior parameters (originating, e.g., from measurement errors) is shown in Fig. 2. In particular, every element of $\hat{x}_{k}$ and $\Sigma_{k}$ was perturbed by Gaussian noise with a standard deviation $\sigma$ of either 0.5 or 1.0. As can be seen from Fig. 2, the reconstructed sequence of residuals tracks the true sequence - allowing for subsequent usage in a fault or change detection algorithm.

\section{CONCLUSION}

In this paper, we have proposed and studied a number of inverse filtering problems for linear Gaussian state-space systems. In particular, we investigated how the sample path of observations, as well as, sensor specifications of a Kalman filtering system can be reconstructed from its posterior distributions over the latent state.

Three nominal problems were considered, and solutions were proposed. The case of noise corrupted posterior distributions (due to, e.g., quantization or measurement errors) was also considered. We showed that in the most general setting, it is possible to reconstruct a signal-to-noise ratio of the sensor, as well as the observations up to a scaling factor. Our results mirror those in [18]: in the most general setting, the discrete HMM parameters were shown to be reconstructible up to permutations, which is analogous to the scaling factor in the continuous setting considered here. Finally, we evaluated the proposed inverse filtering algorithms via numerical simulations.

As future work, we believe it is possible to simplify the proposed solutions, and to extend them to multi-output systems as well as non-autonomous systems, by considering the information filter formulation [4]. We would also like to evaluate the algorithms on data from real-world systems, such as those in [16], [17].

\section{REFERENCES}

[1] V. Krishnamurthy, Partially Observed Markov Decision Processes. Cambridge University Press, 2016.

[2] O. Cappé, E. Moulines, and T. Rydén, Inference in Hidden Markov Models. Springer, 2005.

[3] R. J. Elliott, J. B. Moore, and L. Aggoun, Hidden Markov Models: Estimation and Control. New York, NY: Springer, 1995.

[4] B. D. O. Anderson and J. B. Moore, Optimal Filtering. Prentice-Hall, 1979.

[5] D. Koller and N. Friedman, Probabilistic graphical models: principles and techniques. MIT Press, 2009.

[6] R. E. Kalman, "When is a linear control system optimal," Journal of Basic Engineering, vol. 86, no. 1, pp. 51-60, 1964.

[7] D. Hadfield-Menell, S. J. Russell, P. Abbeel, and A. Dragan, "Cooperative inverse reinforcement learning," in Advances in Neural Information Processing Systems (NIPS'16), 2016.

[8] J. Choi and K.-E. Kim, "Nonparametric Bayesian inverse reinforcement learning for multiple reward functions," in Advances in Neural Information Processing Systems (NIPS'12), 2012.

[9] E. Klein, M. Geist, B. Piot, and O. Pietquin, "Inverse Reinforcement Learning through Structured Classification," in Advances in Neural Information Processing Systems (NIPS'12), 2012.

[10] S. Levine, Z. Popovic, and V. Koltun, "Nonlinear inverse reinforcement learning with Gaussian processes," in Advances in Neural Information Processing Systems (NIPS'11), 2011.

[11] A. Ng, "Algorithms for inverse reinforcement learning," in Proceedings of the 17th International Conference on Machine Learning (ICML'O0), pp. 663-670, 2000.

[12] J. Gertler, Fault Detection and Diagnosis in Engineering Systems. Marcel Dekker, Inc., 1998.

[13] F. Gustafsson, Adaptive filtering and change detection. Wiley, 2000.

[14] F. Gustafsson, "Statistical signal processing approaches to fault detection," Annual Reviews in Control, vol. 31, no. 1, pp. 41-54, 2007.

[15] J. Chen and R. J. Patton, Robust Model-Based Fault Diagnosis for Dynamic Systems. Springer, 1999.

[16] P. Sundvall, P. Jensfelt, and B. Wahlberg, "Fault detection using redundant navigation modules," in Proceedings of the 6th IFAC Symposium on Fault Detection, Supervision and Safety of Technical Processes (SAFEPROCESS'06), vol. 1, pp. 522-527, 2006.

[17] B. Wahlberg and A. C. Bittencourt, "Observers data only fault detection," Proceedings of the 7th IFAC Symposium on Fault Detection, Supervision and Safety of Technical Processes (SAFEPROCESS'09), vol. 42, pp. 959-964, 2009.

[18] R. Mattila, C. R. Rojas, V. Krishnamurthy, and B. Wahlberg, "Inverse filtering for hidden Markov models," in Advances in Neural Information Processing Systems (NIPS'17), pp. 4207-4216, 2017. 